\title{
MANAGING CARE FOR PATIENTS WITH SINONASAL AND ANTERIOR SKULL BASE CANCERS DURING THE COVID-19 PANDEMIC
}

\author{
Mario Turri-Zanoni, $\mathrm{MD}^{1}$ \\ ${ }^{1}$ University of Insubria
}

April 29, 2020

\begin{abstract}
The SARS-CoV-2 pandemic has rapidly transformed healthcare delivery around the globe. Because of the heavy impact of COVID-19 spread, cancer treatments have necessarily been de-prioritized, thus exposing patients to increased risk of morbidity and mortality due to delayed care. In this scenario, cancer specialists need to assess critical oncology patients case by case to carefully balance risk versus benefit in treating tumors and preventing SARS-CoV-2 infection. Here we report early insights into how the management of patients with sinonasal and anterior skull base cancer might be affected by the COVID-19 pandemic. We provide recommendations for preoperative tests, indications for immediate care versus possible delayed treatment, warnings relating to dural resection and intracranial dissection given the potential neurotropism of SARS-CoV2 and practical suggestions for managing cancer care in a period of limited resources. We also postulate some thoughts on the promising role of telemedicine in multidisciplinary case discussions and post-treatment surveillance.
\end{abstract}

Mario Turri-Zanoni, MD; ${ }^{1,2}$ Paolo Battaglia, MD ${ }^{1,2}$ Apostolos Karligkiotis, MD; ${ }^{1}$ Davide Locatelli, MD, ${ }^{2,3}$ Paolo Castelnuovo, MD, FACS, FRCS(Ed). ${ }^{1,2}$

${ }^{1}$ Division of Otorhinolaryngology, Department of Biotechnology and Life Sciences, University of Insubria, Varese, Italy.

${ }^{2}$ Head and Neck Surgery \& Forensic Dissection Research center (HNS\&FDRc), Department of Biotechnology and Life Sciences, University of Insubria, Varese, Italy.

${ }^{3}$ Division of Neurosurgery, Department of Biotechnology and Life Sciences, University of Insubria, Varese, Italy.

SHORT TITLE: Sinonasal cancers and COVID-19

KEYWORDS: Anterior Skull Base; coronavirus; Endoscopic Endonasal Surgery; SARS-CoV-2 viral infection; Sinonasal Malignancy.

\section{COMPLIANCE WITH ETHICAL STANDARDS}

Funding: This was an unfunded study so there is no financial relationship to disclose.

Conflict of Interest: All Authors declare that they have no financial relationships or conflicts of interest to disclose. 
Ethical approval: All procedures performed in studies involving human participants were in accordance with the ethical standards of the institutional and national research committee and with the 1964 Helsinki declaration and its later amendments or comparable ethical standards.

Informed consent: Informed consent was obtained from all individual participants included in the study.

ACKNOWLEDGMENTS. MTZ is a PhD student on the "Biotechnologies and Life Sciences" course at Università degli Studi of Insubria, Varese, Italy.

\title{
CORRESPONDING AUTHOR:
}

\author{
Mario Turri-Zanoni, MD \\ dale di Circolo e Fondazione Macchi \\ Via Guicciardini 9, 21100, Varese, Italy. \\ Phone: (+39) 0332.393278 - Fax: (+39) 0332.393279 \\ E-mail: tzmario@inwind.it
}

Unit of Otorhinolaryngology, Department of Biotechnology and Life Sciences, University of Insubria, Ospe-

\begin{abstract}
The SARS-CoV-2 pandemic has rapidly transformed healthcare delivery around the globe. Because of the heavy impact of COVID-19 spread, cancer treatments have necessarily been de-prioritized, thus exposing patients to increased risk of morbidity and mortality due to delayed care. In this scenario, cancer specialists need to assess critical oncology patients case by case to carefully balance risk versus benefit in treating tumors and preventing SARS-CoV-2 infection. Here we report early insights into how the management of patients with sinonasal and anterior skull base cancer might be affected by the COVID-19 pandemic. We provide recommendations for preoperative tests, indications for immediate care versus possible delayed treatment, warnings relating to dural resection and intracranial dissection given the potential neurotropism of SARS$\mathrm{CoV} 2$ and practical suggestions for managing cancer care in a period of limited resources. We also postulate some thoughts on the promising role of telemedicine in multidisciplinary case discussions and post-treatment surveillance.

Over the past two months, COVID-19 has infected nearly 3,000,000 individuals globally. ${ }^{1}$ After early bewilderment, the scientific community has rallied and put an impressive effort into addressing the needs of the crisis. As a result of these tremendous endeavors, at present, appropriate personal protective equipment (PPE) is more widely available, developments in testing for COVID-19 allow for SARS-CoV-2 detection with RT-PCR within 15 minutes and the sensitivity and specificity of the test is increasing daily thanks to refinements in performing the swabs procedure. ${ }^{2}$ Furthermore, recent studies provide better understanding of the aerosolization risks for different diagnostic and surgical procedures and an evolving body of knowledge is currently available to optimize treatment strategies for COVID-19 patients. ${ }^{3}$
\end{abstract}

However, those now suffering greatly from the COVID-19 pandemic are patients with severe disease such as cancers, who are not only at greater risk of infection from SARS-CoV-2, suffering more severe symptoms given their immunosuppressed status, but may also experience delays in oncological treatments resulting from reallocation of health resources. ${ }^{4}$ This in turn may lead to cancer progression and result in worse survival outcomes.

In this unprecedented scenario, patients affected by sinonasal and anterior skull base cancers are extremely critical and vulnerable for various reasons: their tumor is growing in a deep region in close contact with vital 
neurovascular structures such as brain, dura, carotid artery, orbit, and optic nerve; if not treated quickly, it may rapidly evolve to encroach into these vital areas, leading to severe symptoms such as visual impairment and neurological sequelae. These patients usually present at an advanced stage of disease due to the insidious growth of the tumor with late alarming symptoms, therefore even a short delay in treatment delivery might be fatal, as the patients may became incurable. Such patients are often elderly and multiple comorbidities are generally observed along with an impaired immune status related to the cancer.

It is therefore paramount that criteria are defined to stratify the urgency of cancer care based on patienttailored and cancer-specific factors, while at the same time protecting both patients and healthcare workers from the spread of the pandemic.

The first recommendation is to avoid delay in diagnosis, even in this situation of infectious emergency: contrast-enhanced radiological exams of the head and neck (CT, MRI) as well as systemic assessment of the patient (total body PET-CT scan, neck ultrasounds) should be performed to properly define the extension of the sinonasal cancer; endoscopic-assisted nasal biopsy is essential for histological typing and grading of the tumor. Both these preoperative assessments are mandatory to estimate the patient's prognosis, which is crucial in understanding the intensity of care needed to properly manage any given patient and to balance the risks of potential SARS-CoV-2 infection (immunosuppressive oncological care, frequent hospital attendance) with the negative impact on survival rates of each specific histotype of sinonasal cancers. To perform diagnostic investigations safely, it is mandatory, before any procedures, to define the COVID-19 status of patients, even if they are asymptomatic: nasopharyngeal swabs complemented with chest imaging, ${ }^{5}$ immunological antibodies determination, ${ }^{6}$ and saliva viral load quantification ${ }^{7}$ are used to this purpose.

Secondly, our advice is to undertake regular multidisciplinary tumor board consultations using teleconference systems. Otolaryngologists, neurosurgeons, ophthalmologists, plastic surgeons, radiologists, pathologists, oncologists and radiation therapists can thus coordinate to identify the best level of care for each patient, considering patient-specific and tumor-related factors. Interactions between clinicians involved in the management of sinonasal cancers are necessary for analysis of patients, case by case, to determine a best practice consensus in relation to the local situation (level of the pandemic in region, transport mobility, healthcare resources available). Likewise, multidisciplinary tumor board discussion is paramount in managing care for patients currently receiving treatment (radiotherapy, chemotherapy, immunotherapy) and for those who have recently undergone surgery in order to define the protocol of adjuvant treatments needed.

Third, and most challenging, is to identify patients for whom it would be advisable to defer cancer treatment until after the pandemic. When dealing with early-staged and low-grade sinonasal cancers (e.g. salivary gland tumors, haemangiopericytoma, low-grade sarcoma, low-grade adenocarcinoma), showing an indolent slow-growing pattern, the delay of oncological treatment (e.g. surgery) has a moderate clinically important adverse impact on quality of life and survival rates. Postponement of cancer care is also advisable for patients with multiple high-risk comorbidities, for whom intensified oncological treatments such as major surgery, radiochemotherapy or immunotherapy might severely impact on their immune competence, increasing the risk of COVID-19 related death should they contract the infection. ${ }^{4}$ Patients with sinonasal cancers and active COVID-19 disease should be managed giving the highest priority to treatment of the viral manifestations and deferring the oncological treatments until after complete resolution of the infection. At present, it remains uncertain when the SARS-CoV-2 infection can be considered completely resolved, since negativization of swab specimens and normalization of chest imaging are probably correlated more to the resolution of the acute phase of the disease than to the complete viral clearance from mucosal surfaces and biological fluids such as saliva, urine and stool. ${ }^{8-9}$ In the absence of sound evidence, patients who have recovered from the COVID-19 acute phase should be managed in both outpatient and surgical settings with extra-care in terms of PPE for healthcare workers. ${ }^{10}$

Fourth, and easily understandable, is to provide prompt cancer care to those patients for whom treatments have the potential to cure and which cannot safely be delayed. ${ }^{11}$ Non-surgical treatment with chemo-radiation therapy should be suggested for patients affected by poorly-differentiated sinonasal cancers such as sinonasal undifferentiated carcinoma, neuroendocrine carcinoma, poorly-differentiated squamous cell carcinoma, high- 
grade sarcoma, regardless of the stage of disease at presentation. Patients should be encouraged to receive such treatment in the hospital nearest to their home, in order to avoid long and frequent journeys and to reduce exposure to crowds. Surgery should be proposed for patients presenting in suitable clinical conditions and affected by locally-advanced well-differentiated cancers, or as a salvage treatment after the failure of radiochemotherapy. In patients with close proximity of disease to critical neurovascular structures (dura, periorbit, optic nerve), a delay in surgical treatment would definitively impair the possibility of a cure and result in deterioration of survival outcomes. Surgery should be performed using endoscopic endonasal or combined transcranial/transfacial approaches (orbital exenteration, nasectomy, maxillectomy), based on the local extension of disease and with the goal of free-margins resection, which significantly impacts on the patient prognosis. Healthcare resources should be fully allocated for patients requiring oncological surgery for curative purposes, including intraoperative frozen sections analysis, reconstructive surgery with free-flaps and COVID-free ICU access whenever needed, exactly as before the pandemic. Despite resource constraints, wellselected cases of critical sinonasal cancers should receive appropriate life-saving surgical treatment. Evidence that transcranial surgery might be safer than endonasal approaches, because of the elevated concentration of the SARS-CoV-2 in nose and nasopharynx, is, so far, limited. ${ }^{12}$ Moreover, it has been postulated that small viral particle aerosolization might also be generated from the use of power instruments in contact with blood or bone, not just respiratory mucosa, since, as demonstrated by a recent Chinese series, several biological fluids may contain detectable viral RNA. ${ }^{8}$ However, at present, a more cautious stance should be adopted for endoscopic endonasal approaches as there is a potentially high viral load exposure, given the direct contact with upper respiratory tract mucosa and secretions and the use of power tools which aggressively disrupt potentially virus-containing mucosa. ${ }^{13}$ Therefore, in this setting, it is imperative to maximize the use of PPE for healthcare providers. Another open issue with skull base surgery during the COVID-19 pandemic is related to the potential neurotropism of SARS-CoV-2. New evidence has suggested the possible transcribriform route of the SARS-CoV-2 to the brain, as was reported during the epidemic of SARS-CoV and MERS-CoV which occurred several years ago. ${ }^{14}$ Isolation of SARS-CoV-2 RNA in the cerebrospinal fluid would be conclusive evidence to document the neurovirulence of SARS-CoV-2. Therefore, dura handling during skull base surgery should be performed with particular caution, especially in endoscopic endonasal approaches. Extradural surgery should be advised whenever feasible, with transdural approaches reserved only for selected cases of unavoidable necessity. Other precautionary practices to be adopted in anterior skull base surgery in order to limit the spread of the SARS-CoV-2 infection have been previously described and include: using negative-pressure operating rooms; wearing appropriate PPE, not only during the surgical procedure but also in the pre-operative period (intubation) and during the post-operative care; monitoring the body temperature of patients during the hospitalization period; prohibiting visitors from going to see patients and keeping relatives informed about the patient's medical condition using telephone or video chatting.

The fifth and final recommendation is to promote the use of telemedicine in providing care for sinonasal cancer patients. In this period of pandemic which is threatening the world, many oncology patients are concerned that their needs may be overlooked or marginalized. This is also true for some critical sinonasal cancer patients involved in long-term follow-up, which is generally continued for at least 10 years and, whenever possible, for an individual's lifetime. Video visits represent a valid alternative to in-office evaluations for post-operative surveillance, especially for patients treated many years ago, who are currently under routine yearly or six-monthly follow-up visits, but also for patients treated more recently, who don't strictly need inperson physical evaluation. In all instances, patients should be questioned via telephone or video chat about pending functional issues (nasal breathing, visual ability, lacrimal function, pain) or any new or concerning signs or symptoms that may suggest disease recurrence. Patients requiring post-operative medications, as well as patients with alarming clinical symptoms or radiological signs of cancer recurrence should be visited in person. Adequate preventative measures, in terms of appropriate PPE for healthcare workers, social distancing and body temperature screening for patients and attendants are imperative, especially when performing nasal endoscopy at the outpatient service. ${ }^{15}$

In conclusion, even as we hope to overcome this pandemic, we cannot predict how and when it will end and 
must also be prepared for the likelihood that COVID-19 spread will continue or reappear in different forms. Therefore, the measures proposed here may evolve over time, should be proportional to the degree of risk and take into consideration country-specific resource constraints. It may be that some of the changes forced by this outbreak will permanently transform how we treat cancer in the future. Until then, we will continue to work towards stratifying sinonasal cancers according to urgency, so as to balance prompt oncological treatments with the safety needs of all patients.

\section{REFERENCES}

1. John's Hopkins Center for Systems Science and Engineering. COVID-19 Dashboard by the Center for Systems Science and Engineering (CSSE) at Johns Hopkins University (JHU). https://coronavirus.jhu.edu/map.html. Accessed April 26, 2020.

2. Patel ZM. CORRESPONDANCE: Reflections and new developments within the COVID-19 pandemic. Int Forum Allergy Rhinol 2020; doi: 10.1002/alr.22582.

3. Marini JJ, Gattinoni L. Management of COVID-19 Respiratory Distress. JAMA 2020; doi: 10.1001/jama.2020.6825.

4. Lai SY et al. Changing practice patterns in Head \& Neck Oncologic Surgery in the early COVID-19 era. Authorea. Head \& Neck 2020; doi:10.22541/au.158714509.96811779

5. Miao C, et al. Early chest computed tomography to diagnose COVID-19 from suspected patients: A multicenter retrospective study. Am J Emerg Med 2020; S0735-6757(20)30281-3. doi: 10.1016/j.ajem.2020.04.051.

6. Xiang F, Wang X, He X, Peng Z, Yang B, Zhang J, Zhou Q, Ye H, Ma Y, Li H, Wei X, Cai P, Ma WL. Antibody Detection and Dynamic Characteristics in Patients with COVID-19. Clin Infect Dis 2020; doi: $10.1093 / \mathrm{cid} / \mathrm{ciaa} 461$.

7. Azzi L, et al. Saliva is a reliable tool to detect SARS-CoV-2. J Infect 2020. pii: S0163-4453(20)30213-9. doi:10.1016/j.jinf.2020.04.005.

8. Wang W, Xu Y, Gao R, et al. Detection of SARS-CoV-2 in different types of clinical specimens. JAMA 2020; doi:10.1001/jama.2020.3786.

9. Lan L, Xu D, Ye G, Xia C, Wang S, Li Y, Xu H. Positive RT-PCR Test Results in Patients Recovered From COVID-19. JAMA 2020; doi: 10.1001/jama.2020.2783.

10. Kowalski LP, et al. COVID-19 pandemic: effects and evidence-based recommendations for otolaryngology and head and neck surgery practice. Head \& Neck 2020; doi:10.1002/hed.26164.

11. Coca-Pelaz-A, Takes RP, Hutcheson K et al. Head and Neck Cancer: A review of the impact of treatment delay on outcome. Adv Ther 2018;35(2):153-160.

12. Huang X, Zhu W, Zhao H, Jiang X. In Reply: Precautions for Endoscopic Transnasal Skull Base Surgery During the COVID-19 Pandemic. Neurosurgery 2020; doi: 10.1093/neuros/nyaa145.

13. Workman AD, et al. Endonasal instrumentation and aerosolization risk in the era of COVID-19: simulation, literature review, and proposed mitigation strategies. International Forum of Allergy \& Rhinology 2020; doi:10.1002/alr.22577

14. Li YC, Bai WZ, Hashikawa T. Response to Commentary on "The neuroinvasive potential of SARS-CoV2 may play a role in the respiratory failure of COVID-19 patients". J Med Virol 2020; doi:10.1002/jmv.258244.

15. De Bernardi F, Turri-Zanoni M, Battaglia P, Castelnuovo P. How to reorganize an ENT outpatient service during the COVID-19 outbreak: report from northern Italy. Laryngoscope 2020, doi:10.1002/lary.28716 